\title{
The effect of hospital volume on patient outcomes in severe acute pancreatitis
}

\author{
Hsiu-Nien Shen ${ }^{1,3}$, Chin-Li Lu ${ }^{2,3}$ and Chung-Yi Li, $i^{3,4^{*}}$
}

\begin{abstract}
Background: We investigated the relation between hospital volume and outcome in patients with severe acute pancreatitis (SAP). The determination is important because patient outcome may be improved through volume-based selective referral.
\end{abstract}

Methods: In this cohort study, we analyzed 22,551 SAP patients in 2,208 hospital-years (between 2000 and 2009) from Taiwan's National Health Insurance Research Database. Primary outcome was hospital mortality. Secondary outcomes were hospital length of stay and charges. Hospital SAP volume was measured both as categorical and as continuous variables (per one case increase each hospital-year). The effect was assessed using multivariable logistic regression models with generalized estimating equations accounting for hospital clustering effect. Adjusted covariates included patient and hospital characteristics (model 1), and additional treatment variables (model 2).

Results: Irrespective of the measurements, increasing hospital volume was associated with reduced risk of hospital mortality after adjusting the patient and hospital characteristics (adjusted odds ratio [OR] 0.995, 95\% confidence interval [CI] 0.993-0.998 for per one case increase). The patients treated in the highest volume quartile ( $\geq 14$ cases per hospital-year) had $42 \%$ lower risk of hospital mortality than those in the lowest volume quartile (1 case per hospital-year) after adjusting the patient and hospital characteristics (adjusted OR 0.58, 95\% Cl 0.40-0.83). However, an inverse relation between volume and hospital stay or hospital charges was observed only when the volume was analyzed as a categorical variable. After adjusting the treatment covariates, the volume effect on hospital mortality disappeared regardless of the volume measures.

Conclusions: These findings support the use of volume-based selective referral for patients with SAP and suggest that differences in levels or processes of care among hospitals may have contributed to the volume effect.

Keywords: Severe acute pancreatitis, Hospital volume, Outcomes

\section{Background}

The performance of a hospital is linked to the hospital volume of various surgical procedures and medical conditions [1-6]. Generally, high-volume hospitals have shown to be more efficient and have better outcomes than lowvolume hospitals. Documentation of the volume-outcome relationship is important because patient outcomes may be improved through volume-based selective referral $[1,3]$.

An inverse volume-outcome relationship was revealed in patients with acute pancreatitis (AP) [5,6]. However, prior

\footnotetext{
* Correspondence: cyli99@mail.ncku.edu.tw

${ }^{3}$ Department of Public Health, College of Medicine, National Cheng Kung

University, Tainan, Taiwan

${ }^{4}$ Department of Public Health, College of Public Health, China Medical University, Taichung, Taiwan

Full list of author information is available at the end of the article
}

studies suffered from shortcomings that may have overestimated the volume effect and limited the generalization of findings [5-7]. These shortcomings included failures to exclude readmissions or recurrences [5,6], consider the severity of AP [5], account for the hospital clustering effect [6], and model hospital volume as a continuous variable $[5,6,8,9]$. Besides, the definition of high volume hospitals considerably differed between the studies $(\geq 118$ cases/year vs. $>16$ cases/9 months) $[5,6]$, which limited the practical application of the results. Moreover, the inclusion of both mild and severe cases in these studies [5,6] implied that the results were generalized to all AP patients, which was unreasonable because selective referral was not justified for mild and self-limited diseases, such as mild AP [10,11]. Therefore, we conducted this study and enrolled only

\section{Biomed Central}


severe cases from a national cohort of patients with firstattack AP [12] to investigate the effect of hospital volume on patient outcomes.

\section{Methods \\ Database}

Data regarding the patients were obtained from the Taiwan National Health Insurance Research Database (NHIRD), which is released for research purposes by the National Health Research Institute and is one of the largest and most comprehensive databases in the world $[12,13]$. Information included in the inpatient database included sex, date of birth, encrypted patient identification numbers, residential or work area, dates of admission and discharge, medical institutions providing services, the International Classification of Diseases, Ninth Revision, Clinical Modification (ICD-9-CM) codes of diagnoses (up to five) and procedures (up to five), outcome at hospital discharge (recovered, died or transferred), order codes and hospital charges. The study was exempt from obtaining approval from the Human Subjects Institutional Review Board and informed consent from the patients due to the use of an encrypted administrative database.

\section{Definitions and patients}

AP was defined by ICD-9-CM code 577.0 in any position of the five diagnoses [12]. Severe AP (SAP) was defined primarily according to the Atlanta classification scheme [14], but was modified by the presence of intensive care unit (ICU) admission (as a surrogate of acute physiology and chronic health evaluation [APACHE] II score $\geq 8$ ), organ dysfunction or failure, gastrointestinal bleeding, or local complications [12,13]. The enrollment of the patients is shown in Figure 1. We excluded patients $(n=1,414)$ with biliary AP who received cholecystectomy and intensive care and had no organ failure, gastrointestinal bleeding, or local complications because these patients may have had mild biliary AP and received ICU care only after the surgery [12]. After exclusion, we enrolled 22,551 patients in the following analysis.

\section{Exposure variable}

The annual number of SAP cases per hospital was the main exposure variable. The distribution of hospital volume versus hospital mortality per hospital-year is shown in Figure 2.

We first measured hospital volume as a continuous variable (per 1 case increase per hospital-year) to assess the effect of hospital volume on outcomes [8]. Then, the hospital volume was sorted and divided into 4 and 9 about-equal subsets, respectively, to help visualize the effect of increasing volume and for practical uses. The quartile ranges were $1,2-5,6-13$ and $\geq 14$ SAP cases per hospital-year, respectively. The 9-quantile ranges (corresponding proportion of hospital-year units) were 1 (22.0\%), 2 (12.2\%), 3 (8.2\%), 4-5 (10.4\%), 6-8 (11.1\%), 912 (10.1\%), 13-19 (10.7\%), 20-33 (9.3\%), and $\geq 34$ (5.9\%) SAP cases per hospital-year, respectively. Patients were allocated essentially in decentile except that the first two decentiles with 1 case per hospital-year were collapsed into one subset due to the skewed nature of the volume. The quartiles were used for both presentation and comparison of the results and the 9-quantiles were used primarily to show the trend of volume effect on hospital mortality.

\section{Covariates}

Two levels of covariates, baseline and additional, were included. The baseline covariates were patient and hospital characteristics. The patient characteristics included age, sex, year of admission, urbanization (urban, suburban and rural area) [15], Charlson comorbidity index $[16,17]$, and causes (biliary, alcohol-related, both or others) and severity criteria of AP.

The hospital characteristics included hospital level (medical center [ $>500$ beds], regional [250-500 beds] and district hospitals [20-249 beds]) [13], hospital ownership (public, private not-for-profit, or private forprofit) [18] and geographical location (northern, central, southern, and eastern Taiwan).

Additional covariates were employed to account for the process of care and included the following selected treatments: cholecystectomy and life-support measures (including total parenteral nutrition [TPN], hemodialysis, vasopressors and mechanical ventilation [MV]) [12].

\section{Outcomes}

Primary outcome was hospital mortality [12]. Secondary outcomes were hospital charges and hospital length of stay (LOS). The charges were adjusted to the 2009 price levels in United States Dollars (USD) [12].

\section{Statistics}

Data were analyzed using the SAS software, version 9.1 (SAS Institute, Inc., Cary, NC, USA). Continuous variables are presented as median (interquartile range, IQR); discrete ones as count or percentage. A two-tailed $\mathrm{p}$ value of $<0.05$ was considered significant.

We hypothesized that hospital volume is inversely associated with hospital LOS, charges and mortality. We assumed that the relationship is linear. In the univariate analysis, we performed ANOVA test for the linearity of scaled variables and linear-by-linear association Chi-square test for categorical data. To account for clustering, the effect of hospital volume was analyzed by using regression model with generalized estimating equations methods [19], specifying an exchangeable structure of a working correlation matrix, to construct regression models. Hospital 


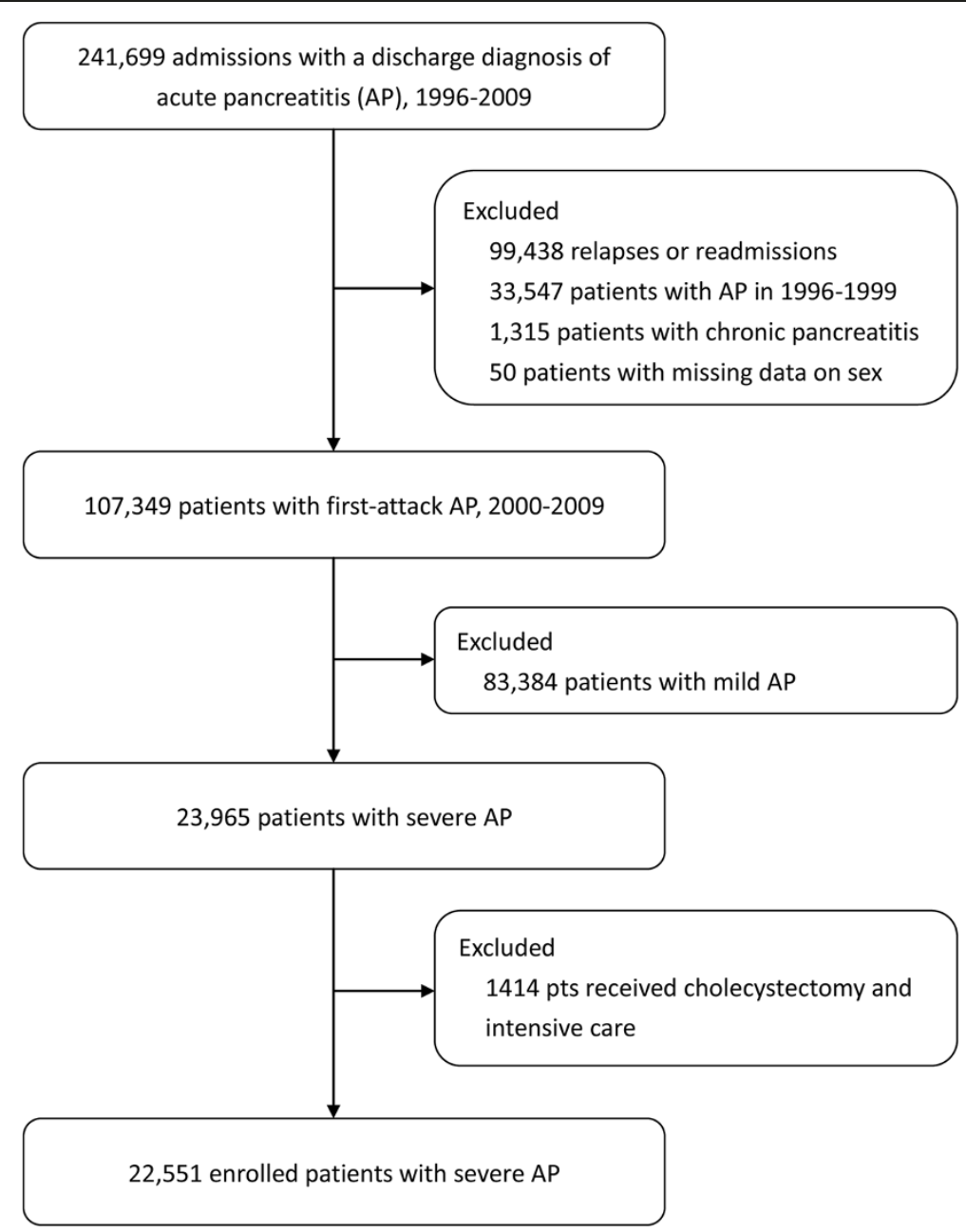

Figure 1 Study flow diagram. (Note: Patients hospitalized for AP between 1996 and 1999 were excluded to ensure the inclusion of first-attack cases because most relapses occur within the first 4 years after the first "attack)."

mortality was regressed with a logit link function and hospital LOS and charges were log-transformed and then regressed with a linear link function on hospital volume. Both univariable and multivariable analyses were performed to yield the crude and adjusted estimates. In the multivariable analysis, we performed two consecutive models adjusting for the baseline covariates in model 1 and for the baseline and additional covariates in model 2 . We examined the volume effect by first using hospital volume as a continuous variable and then as a categorical variable, as aforementioned. The effects of hospital volume are presented as odds ratios (OR) with 95\% confidence interval (CI) for hospital mortality, and as percentage changes with $95 \% \mathrm{CI}$, which were calculated from the exponentiated regression coefficients minus 1 [20], for hospital LOS and charges. Model performance was assessed by using R-squared and c statistics. The variance of outcomes explained by hospital volume was assessed and compared with that of other covariates by using the coefficient of determination $\left(r^{2}\right)$ for hospital LOS and charges and, by the percentage change of -2 log likelihood (-2LL) for hospital mortality. The change in -2LL (\%) was calculated by dividing the difference in -2LL values between the univariable and the intercept-only models by the corresponding value of the intercept-only model. The $r^{2}$ was derived from the univariable linear regression model. We examined the estimated slope coefficients and the standard errors of the mean and found no indication of collinearity.

\section{Results}

\section{Hospital and patient characteristics}

Table 1 shows the characteristics of the SAP patients. Hospital volume was correlated with hospital level, ownership and geographic location. Low quartile hospitals tended to be at the district level, private-for-profit ownership, and located in southern Taiwan, whereas higher quartile ones tended to be at the regional or center level, public or private not-for-profit ownership and located in the north. 


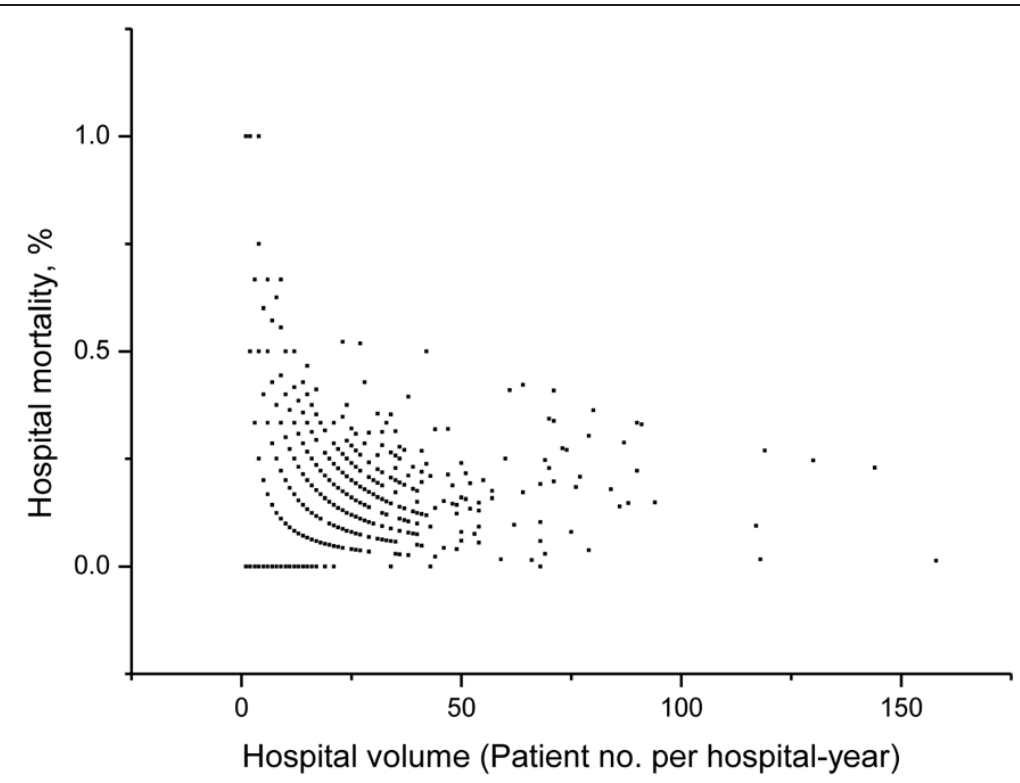

Figure 2 Distribution of hospital volume versus hospital mortality per hospital-year in severe acute pancreatitis (Note: There were 467 hospitals contributing to a total of $\mathbf{2 , 2 0 8}$ hospital-years. Median hospital volume was 5 cases per hospital-year [interquartile range $2-13]$ ).

More than two-thirds of the patients were treated in the highest quartile hospitals, and the proportion increased over time. With increasing hospital volume, patients tended to be older and less male-predominance, to live in urban areas and to have more complex comorbidities (Charlson-Comorbidity Index $\geq 3$ ) and biliary causes. Among severity criteria of AP, organ failure was the most common, but the distributional pattern of the individual

Table 1 Characteristics of hospitals and patients with severe acute pancreatitis $(n=22,551)$

\begin{tabular}{|c|c|c|c|c|c|}
\hline \multirow[t]{2}{*}{ Variables } & \multicolumn{4}{|c|}{ Hospital volume quartiles(by case No. per hospital-year) } & \multirow{2}{*}{$\begin{array}{l}P \\
\text { values } \\
\text { for } \\
\text { trend }\end{array}$} \\
\hline & 1 & $2-5$ & 6-13 & $14+$ & \\
\hline \multicolumn{6}{|l|}{ Hospital characteristics } \\
\hline No. of hospital-years & 486 & 679 & 514 & 529 & - \\
\hline No. of hospitals & 276 & 261 & 165 & 104 & - \\
\hline Hospital level, \% & & & & & $<0.001$ \\
\hline Medical center & 0 & 0.5 & 5.0 & 39.1 & \\
\hline Regional hospital & 4.5 & 16.8 & 46.7 & 48.1 & \\
\hline District hospital & 95.5 & 82.7 & 48.3 & 12.8 & \\
\hline Hospital ownership, \% & & & & & $<0.001$ \\
\hline Private (for-profit) & 70.3 & 53.2 & 32.9 & 17.8 & \\
\hline Private (not-for-profit) ${ }^{*}$ & 10.8 & 17.6 & 33.9 & 50.7 & \\
\hline Public & 18.9 & 29.2 & 33.2 & 31.5 & \\
\hline Geographic location, \% & & & & & $<0.001$ \\
\hline Northern & 24.5 & 36.2 & 39.6 & 37.4 & \\
\hline Central & 29.8 & 26.5 & 28.4 & 28.3 & \\
\hline Southern & 39.7 & 32.4 & 25.1 & 29.5 & \\
\hline Eastern & 6.0 & 4.9 & 6.9 & 4.7 & \\
\hline Patient No. by year of admission & & & & & $<0.001$ \\
\hline $2000-2001$ & 129 & 578 & 929 & 2150 & \\
\hline $2002-2003$ & 102 & 410 & 1037 & 2689 & \\
\hline
\end{tabular}


Table 1 Characteristics of hospitals and patients with severe acute pancreatitis $(n=\mathbf{2 2 , 5 5 1 )}$ (Continued)

\begin{tabular}{|c|c|c|c|c|c|}
\hline 2004-2005 & 95 & 400 & 953 & 3520 & \\
\hline 2006-2007 & 89 & 379 & 873 & 3544 & \\
\hline 2008-2009 & 71 & 338 & 803 & 3462 & \\
\hline \multicolumn{6}{|l|}{ Patient characteristics } \\
\hline Median age (IQR), yr & $50(38-70)$ & $52(39-72)$ & $54(41-73)$ & $57(42-73)$ & $<0.001$ \\
\hline Male sex, \% & 75.1 & 72.4 & 68.0 & 64.9 & $<0.001$ \\
\hline Urbanization, \% & & & & & 0.039 \\
\hline Urban & 45.8 & 49.8 & 49.9 & 50.8 & \\
\hline Suburban & 37.7 & 34.6 & 35.7 & 34.6 & \\
\hline Rural & 16.6 & 15.6 & 14.3 & 14.6 & \\
\hline Charlson Comorbid Index, \% & & & & & 0.688 \\
\hline 0 & 15.6 & 20.6 & 21.1 & 24.9 & \\
\hline 1 & 47.1 & 42.9 & 42.8 & 37.0 & \\
\hline 2 & 24.9 & 23.4 & 20.8 & 21.5 & \\
\hline$\geq 3$ & 12.3 & 13.1 & 15.3 & 16.5 & \\
\hline Causes, \% & & & & & $<0.001$ \\
\hline Biliary stone & 10.3 & 15.0 & 16.6 & 17.1 & \\
\hline Alcohol abuse & 4.1 & 3.5 & 4.0 & 4.1 & \\
\hline \multicolumn{6}{|l|}{ Severity criteria } \\
\hline ICU admission & 24.5 & 37.1 & 45.4 & 49.7 & $<0.001$ \\
\hline Organ failure & 48.4 & 51.6 & 52.2 & 60.1 & $<0.001$ \\
\hline Gastrointestinal bleeding & 42.2 & 37.5 & 33.2 & 27.5 & $<0.001$ \\
\hline Local complications & 3.7 & 4.2 & 7.1 & 7.0 & $<0.001$ \\
\hline \multicolumn{6}{|l|}{ Treatments, \% } \\
\hline Cholecystectomy $^{\dagger}$ & 1.0 & 1.0 & 1.6 & 1.9 & 0.001 \\
\hline Total parenteral nutrition & 1.9 & 2.7 & 6.7 & 12.7 & $<0.001$ \\
\hline Vasopressors & 14.8 & 18.2 & 21.3 & 26.1 & $<0.001$ \\
\hline Hemodialysis & 4.5 & 4.2 & 7.4 & 9.7 & $<0.001$ \\
\hline Mechanical ventilation & 17.5 & 18.2 & 22.4 & 30.0 & $<0.001$ \\
\hline Median hospital LOS (IQR), d & $6(4-13)$ & $7(3-13)$ & $8(4-16)$ & $10(5-20)$ & $<0.001$ \\
\hline $\begin{array}{l}\text { Median hospital charges } \\
\text { (IQR), USD }\end{array}$ & 515 (284-1736) & 713 (354-1845) & $1069(510-2814)$ & 1715 (713-4619) & 0.044 \\
\hline Hospital mortality, \% & 12.8 & 12.2 & 14.2 & 16.6 & $<0.001$ \\
\hline
\end{tabular}

IQR: interquartile range; ICU: intensive care unit; LOS: length of stay; USD: United States dollars.

*A private not-for-profit hospital is a tax-exempt, commercial and entrepreneurial organization, which operates roughly in the same way with a private for-profit hospital except the difference in missions and goals on providing community benefit services [18].

${ }^{\dagger}$ The surgery was performed during admission for first-attack acute pancreatitis.

severity criteria differed. As hospital volume increased, the prevalence fell in gastrointestinal bleeding, but increased in ICU admission, organ failure and local complication.

More patients received TPN, vasopressors, hemodialysis, and MV in higher volume hospitals. Cholecystectomy was rarely performed in SAP and tended to be done in higher volume hospitals.

\section{Hospital volume-outcome relationship}

The crude estimates showed that median hospital LOS and charges increased with hospital volume. A similar trend was observed for hospital mortality except that the mortality slightly fell in the second volume quartile (Table 1).

When hospital volume was entered into the regression models as a continuous variable (Table 2), the unadjusted effect of volume on hospital mortality was not statistically significant. Model 1 shows that hospital volume was inversely associated with hospital mortality. After controlling for additional treatment-related covariates, the volume effect on hospital mortality attenuated and became insignificant (Model 2). Volume, as a continuous variable, appeared to have no effect on hospital LOS, but 
Table 2 Effects of hospital volume (as a continuous variable) on outcomes in patients with severe acute pancreatitis $(n=22,551)$

\begin{tabular}{lllc}
\hline Outcomes & $\begin{array}{l}\text { Crude OR or percent } \\
\text { change }(\mathbf{9 5 \%} \text { Cl) }\end{array}$ & \multicolumn{2}{c}{ Adjusted OR or percent change (95\% Cl) } \\
\cline { 3 - 4 } & $-0.08 \%(-0.28 \%, 0.12 \%)$ & $-0.06 \%(-0.25 \%, 0.13 \%)$ & Model 2 $^{\dagger}$ \\
\hline Hospital LOS & - & $22.74 \%$ & $-0.10 \%(-0.26 \%, 0.05 \%)$ \\
R-squared & $-0.17 \%(-0.32 \%,-0.01 \%)$ & $-0.14 \%(-0.33 \%, 0.06 \%)$ & $30.31 \%$ \\
Hospital charges & - & $53.92 \%$ & $-0.16 \%(-0.27 \%,-0.04 \%)$ \\
R-squared & $-0.999(0.994,1.003)$ & $0.995(0.993,0.998)$ & $64.16 \%$ \\
Hospital mortality & - & $85.4 \%$ & $0.999(0.996,1.002)$ \\
C statistic & & $93.0 \%$ \\
\hline
\end{tabular}

OR: odds ratio; $\mathrm{Cl}$ : confidence interval; LOS: length of stay.

* Covariates in model 1 included age (as a continuous variable), sex, year of admission, Charlson-Comorbidity Index (categorized as 0,1,2 and $\geq 3$ ), urbanization, hospital level, the ownership of hospital, the region of hospital, causes of acute pancreatitis (categorized into biliary, alcohol-related, both or others), intensive care unit admission, organ failure, gastrointestinal bleeding and local complication.

${ }^{+}$Model 2 enrolled all covariates of model 1 and additional treatment covariates, including cholecystectomy, total parenteral nutrition, vasopressors, hemodialysis and mechanical ventilation.

was inversely associated with lower hospital charges in the unadjusted and fully adjusted models.

When hospital volume was entered into the regression models as a categorical variable (Table 3 ), the results of the volume effect on hospital mortality were similar to those modeled using volume as a continuous variable. Compared to the lowest quartile hospitals, SAP patients treated in higher volume hospitals had

Table 3 Effects of hospital volume (as a categorical variable) on outcomes in patients with severe acute pancreatitis $(n=22,551)$

\begin{tabular}{|c|c|c|c|c|}
\hline \multirow[t]{2}{*}{ Outcomes } & \multirow{2}{*}{$\begin{array}{l}\text { Hospital } \\
\text { volume }\end{array}$} & \multirow{2}{*}{$\begin{array}{l}\text { Crude OR or percent } \\
\text { change }(95 \% \mathrm{Cl})\end{array}$} & \multicolumn{2}{|c|}{ Adjusted OR or percent change $(95 \% \mathrm{Cl})$} \\
\hline & & & Model $1^{*}$ & Model $2^{+}$ \\
\hline \multicolumn{5}{|c|}{ Hospital LOS } \\
\hline & Quartile 1 & Ref. & Ref. & Ref. \\
\hline & Quartile 2 & $-2.52 \%(-12.78 \%, 8.94 \%)$ & $-15.64 \%(-24.13 \%,-6.21 \%)$ & $-12.95 \%(-21.22 \%,-3.82 \%)$ \\
\hline & Quartile 3 & $0.00 \%(-11.15 \%, 12.56 \%)$ & $-16.78 \%(-25.57 \%,-7.02 \%)$ & $-14.95 \%(-23.46 \%,-5.50 \%)$ \\
\hline & Quartile 4 & $0.00 \%(-4.50 \%, 23.62 \%)$ & $-16.36 \%(-25.05 \%,-6.05 \%)$ & $-14.80 \%(-23.84 \%,-4.70 \%)$ \\
\hline & $P$ for trend & 0.909 & 0.005 & 0.007 \\
\hline & R-squared & - & $23.81 \%$ & $30.36 \%$ \\
\hline \multicolumn{5}{|c|}{ Hospital charges } \\
\hline & Quartile 1 & Ref. & Ref. & Ref. \\
\hline & Quartile 2 & $8.65 \%(-4.50 \%, 23.62 \%)$ & $-9.99 \%(-18.65 \%,-0.41 \%)$ & $-5.15 \%(-13.38 \%, 3.86 \%)$ \\
\hline & Quartile 3 & $7.39 \%(-7.63 \%, 24.85 \%)$ & $-10.03 \%(-19.18 \%, 0.14 \%)$ & $-5.60 \%(-14.28 \%, 3.96 \%)$ \\
\hline & Quartile 4 & $9.25 \%(-6.79 \%, 28.05 \%)$ & $-11.42 \%(-20.90 \%,-0.80 \%)$ & $-6.79 \%(-15.92 \%, 3.33 \%)$ \\
\hline & P for trend & 0.398 & 0.053 & 0.205 \\
\hline & R-squared & - & $53.89 \%$ & $64.16 \%$ \\
\hline \multicolumn{5}{|c|}{ Hospital mortality } \\
\hline & Quartile 1 & Ref. & Ref. & Ref. \\
\hline & Quartile 2 & $0.98(0.73,1.31)$ & $0.58(0.42,0.82)$ & $0.75(0.49,1.15)$ \\
\hline & Quartile 3 & $1.09(0.83,1.45)$ & $0.62(0.45,0.88)$ & $0.83(0.54,1.26)$ \\
\hline & Quartile 4 & $1.02(0.77,1.35)$ & $0.58(0.40,0.83)$ & $0.75(0.49,1.16)$ \\
\hline & $P$ for trend & 0.720 & 0.012 & 0.281 \\
\hline & c statistic & - & $85.4 \%$ & $93.0 \%$ \\
\hline
\end{tabular}

OR: odds ratio; $\mathrm{Cl}$ : confidence interval; LOS: length of stay.

* Covariates in model 1 included age (as a continuous variable), sex, year of admission, Charlson-Comorbidity Index (categorized as 0,1,2 and $\geq 3$ ), urbanization, hospital level, the ownership of hospital, the region of hospital, causes of acute pancreatitis (categorized into biliary, alcohol-related, both or others), intensive care unit admission, organ failure, gastrointestinal bleeding and local complication.

${ }^{\dagger}$ Model 2 enrolled all covariates of model 1 and additional treatment covariates, including cholecystectomy, total parenteral nutrition, vasopressors, hemodialysis and mechanical ventilation. 


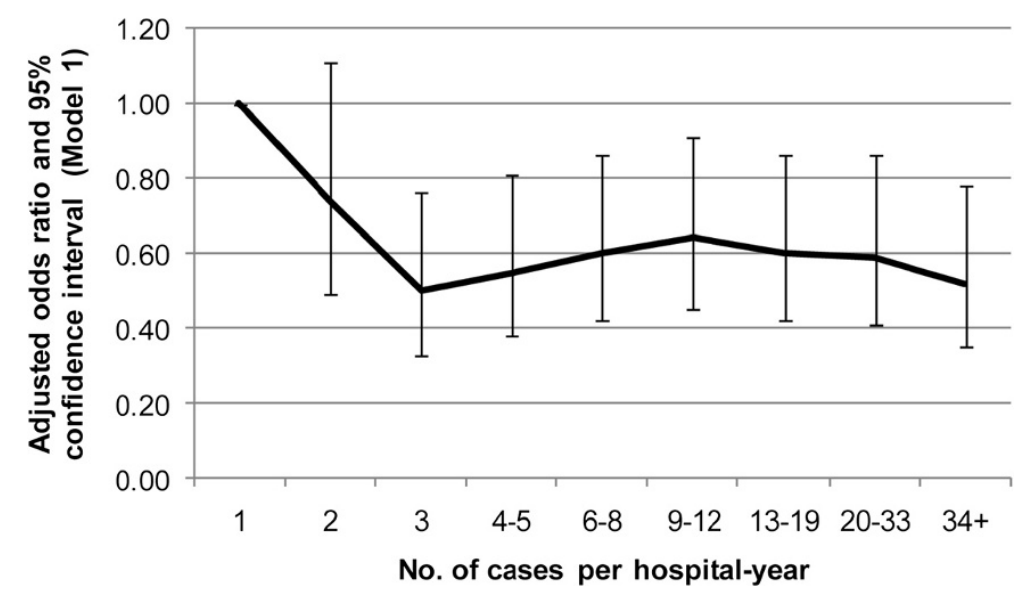

Figure 3 Effect of hospital volume on hospital mortality in severe acute pancreatitis adjusting for patient and hospital characteristics (Note: Hospital volume was divided into 9 about-equal subsets).

nearly $40 \%$ less risk of hospital mortality (Model 1 ). Volume effect became insignificant when differences in treatment among volume quartiles were controlled. When hospital volume was divided into 9 about-equal subsets, the effect of volume on hospital mortality appeared to plateau at $\geq 3$ SAP cases per year (Figure 3). However, the results of hospital LOS and charges differed from those using volume as a continuous variable. For example, compared to the lowest quartile hospitals, patients treated in higher volume hospitals had a shorter hospital stay after controlling for the baseline covariates (Model 1). The volume effect on hospital LOS attenuated but persisted after additional adjustment of the treatment covariates (Model 2). Conversely, the volume effect on hospital charges was significant only after controlling for the baseline covariates (Model 1).

The variances in the outcomes explained by various variables are shown in Figure 4. The extent of the effect of hospital volume was greater on hospital mortality than on hospital LOS and charges. Nevertheless, the degree of the effect associated with hospital volume on various outcomes was relatively small compared to those of most patient and hospital characteristics.

\section{Discussion}

In this study, we found that hospital SAP volume, either as a continuous or as a categorical variable, was inversely associated with hospital mortality after controlling for the baseline covariates. The volume effect disappeared when the differences in treatment among hospitals were controlled, suggesting that the effect can be largely explained by the different levels or processes of care across hospitals. Although the results on hospital LOS and charges were somewhat dependent on the definition of volume measures, trend analyses suggested that higher hospital volume appeared to be associated with shorter stay and lower cost.

We found that SAP patients treated in higher volume hospitals had nearly $40 \%$ less risk of hospital mortality than the lowest volume quartile ones. A recent report from Japan showed an even greater risk reduction in high volume hospitals [6]. In the report, Murata and coworkers [6] analyzed 7,007 patients with AP, including 662 severe cases, in 776 hospitals recruited over a 9month period and found that in-hospital mortality (within 30 days) was about 66\% lower for severe cases in high volume hospitals (defined as $>16$ AP cases) than in low volume ones ( $<10$ AP cases). Had they limited their analysis to the volume of SAP cases and extended the study period to one year, the cutoff point for the lowest volume category (about 1 SAP case/year) would be very close to our results. However, in addition to working with a relatively small sample size, they also failed to exclude readmissions or recurrences of AP, and did not account for the hospital clustering effect in the analysis. Therefore, the effects are likely to be overinflated [21]. Besides, they defined SAP only as a dichotomous variable, which may have limited severity adjustment. Moreover, similar to an earlier study in the United States [5], they included both mild and severe cases in the analysis, which implied that volume-based selective referral, if adopted, would be applied to all patients with AP. However, some potential disadvantages of the volume-based policy [11] make us believe that selective referral should be limited to high risk patients, such as SAP cases. The transfer to a distant high-volume hospital is unreasonable for a mild AP patient who would recover within several days without the need of specific treatment other than simple supportive care [10].

The beneficial effects associated with high hospital SAP volume are likely related to the overall experience 

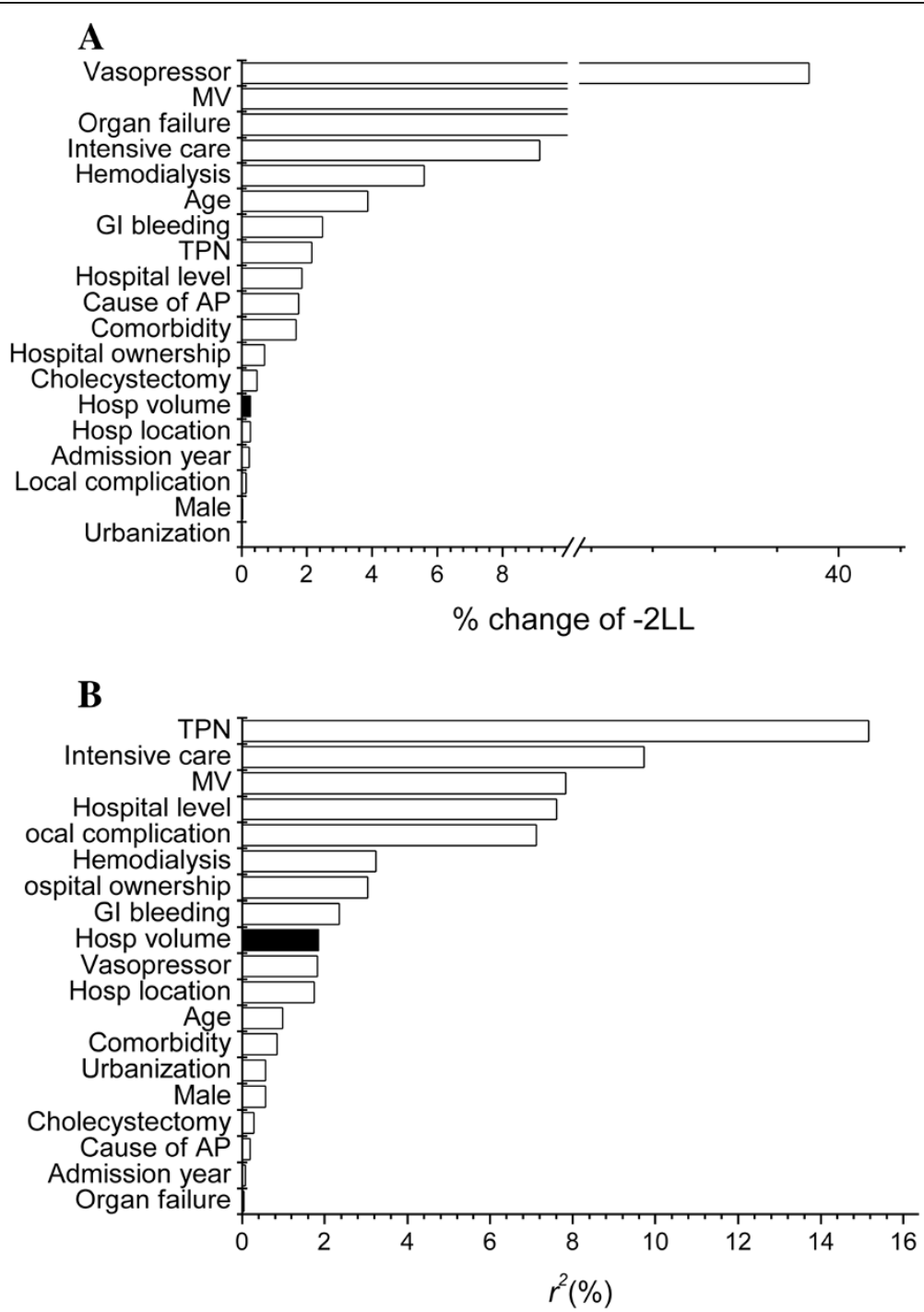

C

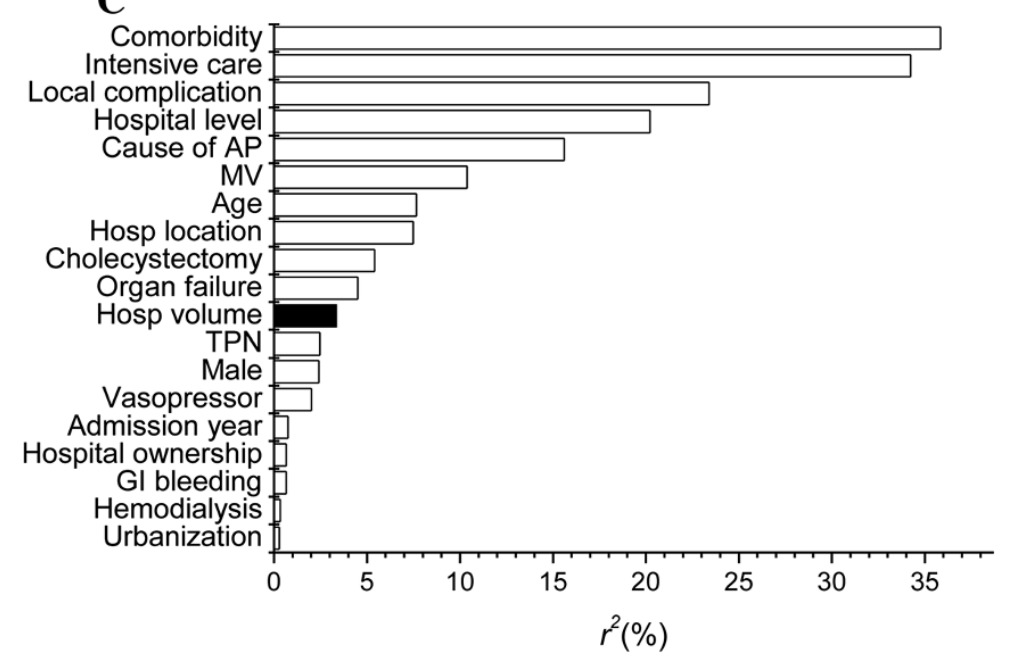

Figure 4 Variances of outcomes (A: hospital mortality, B: hospital length of stay, C: hospital charges) explained by various variables (MV: mechanical ventilation; TPN: total parenteral nutrition; AP: acute pancreatitis). 
of the health care team, the processes of care and some organizational features, which included the availability of specialists, interventions and intensive care in complicated cases $[5,11,22]$. We found that the volume effect in SAP patients disappeared after additional adjustment of cholecystectomy and life-support measures, indicating that different levels or processes of care may have contributed to the observed effect. This finding is interesting and novel because previous studies did not consider the variation of treatment regimens across hospitals $[5,6]$, which may be a probable mechanism for the observed relationship between volume and outcome. High volume hospitals are likely to have more specialists, interventions, and advanced intensive cares that are critical in saving the lives of pancreatitis patients. Moreover, we found that hospital mortality plateaued at $\geq 3$ cases/year, suggesting a possible threshold effect of hospital volume on SAP outcome. The threshold effect may also be responsible for the insignificant association between hospital volume, as continuous variable, and hospital LOS and charges. Because the number of cases per year was within the margin of error of other values (Figure 3), the threshold value requires further validation.

This present study has important implications on the health policy and future research for the treatment of pancreatitis in Taiwan. Our recent study shows that hospital charges per patient with acute pancreatitis in Taiwan increased by nearly 50\% from 2000 to 2009 [12]. Most of the increase was likely due to the lack of a formal referral system and an improper reimbursement policy. In Taiwan, reimbursement for some services (e.g., physician staffing and ICU bed) increases with hospital levels, which has promoted the growth of higher level hospitals out of proportion to lower level hospitals [23]. Consequently, patients with mild pancreatitis who could be treated properly in lower level hospitals usually sought medical care in higher level hospitals, leading to an overall higher cost of health care for pancreatitis. The reimbursement policy may also help explain why hospital volume did not affect hospital charges in this study because most hospitals with lower quartile volumes were lower level hospitals (i.e., district hospitals) and most of the hospitals with higher quartile volumes were higher level hospitals (i.e., regional hospitals or medical centers). The findings in the present study support the need for a better referral system that can limit the access of patients with mild pancreatitis and transfer severe cases to high-volume hospitals in Taiwan, which may lead to appropriate reallocation of medical resources. However, further research is needed to examine the outcome of transfer and the cost-effectiveness of the volumebased selective referral.

Some limitations deserve comments. First, the definition of SAP in this study tended to include patients who had a more severe attack and had received intensive care and/or invasive treatments. For example, some patients might not be included if they had an APACHE II score $\geq 8$ but were cared for only outside an ICU or if they had local complications but did not receive invasive procedures. Besides, some patients with organ failure may also be missed because of the limited number of diagnostic codes. However, the selection of a more severe group of patients is non-differential among hospitals, which tends to bias the observed effect towards the null. Second, residual confounding may be present because adequate adjustment for potential confounders may be lacking, which is especially true for biliary and alcohol-related AP. The potential bias arising from residual confounding is uncertain. Finally, the generalizability of the findings may be limited by the different prevalence of causes in other regions of the world because the hospital volume-outcome relationship may be more important for biliary causes (e.g., expertise in ERCP) of pancreatitis as compared to alcoholic causes.

\section{Conclusions}

The results of this study support the use of volumebased selective referral for patients with SAP. The volume threshold was rather low (i.e., 3 cases/year) and would not pose a significant caseload for current high volume hospitals. The outcomes of the transfer of SAP patients to high volume hospitals and its cost-effectiveness require further research.

\section{Abbreviations}

-2LL: -2 log likelihood; AP: Acute pancreatitis; APACHE II score: Acute physiology and chronic health evaluation II score; Cl: Confidence interval; ICD-9-CM: International classification of diseases ninth revision, clinical modification; ICU: Intensive care unit; IQR: interquartile range; LOS: Length of stay; MV: Mechanical ventilation; OR: Odds ratio; NHIRD: National health insurance research database; SAP: Severe acute pancreatitis; TPN: Total parenteral nutrition; USD: United States dollar.

\section{Competing interests}

The authors declare that they have no competing interests.

\section{Authors' contributions}

HNS designed the study, obtained funding, performed data mining and processing, did statistical analyses, drafted the initial manuscript, and revised important content. CLL contributed to the study design, data mining and processing, analyses and interpretation of results, and revision for important content. CYL participated in the interpretation of results and revision for important content. All authors read and approved the final manuscript.

\section{Acknowledgments}

The study was performed in Chi Mei Medical Center and supported by grant CMFHR10025 and CMFHR10136 from the hospital. The study was based in part on data from the National Health Insurance Research Database provided by the Bureau of National Health Insurance, Department of Health and managed by National Health Research Institutes. The interpretation and conclusions contained herein do not represent those of Bureau of National Health Insurance, Department of Health or National Health Research Institutes. 


\section{Author details}

${ }^{1}$ Department of Intensive Care Medicine, Chi Mei Medical Center, No. 901 Chung-Hwa Road, Yong-Kang City, Tainan, Taiwan. ²Department of Medical Research, Chi Mei Medical Center, No. 901 Chung-Hwa Road, Yong-Kang City, Tainan, Taiwan. ${ }^{3}$ Department of Public Health, College of Medicine, National Cheng Kung University, Tainan, Taiwan. ${ }^{4}$ Department of Public Health, College of Public Health, China Medical University, Taichung, Taiwan

Received: 4 April 2012 Accepted: 17 July 2012

Published: 17 August 2012

\section{References}

1. Epstein AM: Volume and outcome-it is time to move ahead. N Engl J Med 2002, 346:1161-1164.

2. Halm EA, Lee C, Chassin MR: Is volume related to outcome in health care? A systematic review and methodologic critique of the literature. Ann Intern Med 2002, 137:511-520.

3. Kahn JM, Linde-Zwirble WT, Wunsch H, Barnato AE, Iwashyna TJ, Roberts MS, Lave JR, Angus DC: Potential value of regionalized intensive care for mechanically ventilated medical patients. Am J Respir Crit Care Med 2008, 177:285-291.

4. Glance LG, Li Y, Osler TM, Dick A, Mukamel DB: Impact of patient volume on the mortality rate of adult intensive care unit patients. Crit Care Med 2006, 34:1925-1934.

5. Singla A, Simons J, Li YF, Csikesz NG, Ng SC, Tseng JF, Shah SA: Admission volume determines outcome for patients with acute pancreatitis. Gastroenterology 2009, 137:1995-2001.

6. Murata A, Matsuda S, Mayumi T, Yokoe M, Kuwabara K, Ichimiya Y, Fujino Y, Kubo T, Fujimori K, Horiguchi $\mathrm{H}$ : Effect of hospital volume on clinical outcome in patients with acute pancreatitis, based on a national administrative database. Pancreas 2011, 40:1018-1023.

7. Wu BU: The impact of hospital volume on outcomes in acute pancreatitis: a case for centers of excellence? Gastroenterology 2009, 137:1886-1888.

8. Livingston $\mathrm{EH}, \mathrm{CaO} \mathrm{J}$ : Procedure volume as a predictor of surgical outcomes. JAMA 2010, 304:95-97.

9. Altman DG, Lausen B, Sauerbrei W, Schumacher M: Dangers of using "optimal" cutpoints in the evaluation of prognostic factors. J Nat/ Cancer Inst 1994, 86:829-835.

10. Banks PA, Freeman ML: Practice guidelines in acute pancreatitis. Am J Gastroenterol 2006, 101:2379-2400.

11. Shahian DM, Normand SL: The volume-outcome relationship: from Luft to Leapfrog. Ann Thorac Surg 2003, 75:1048-1058.

12. Shen $\mathrm{HN}$, Lu CL, Li CY: Epidemiology of first-attack acute pancreatitis in Taiwan from 2000 through 2009: a nationwide population-based study. Pancreas (publish ahead of print) 2012, 41:696-702.

13. Shen HN, Lu CL: Incidence, resource use, and outcome of acute pancreatitis with/without intensive care: a nationwide population-based study in Taiwan. Pancreas 2011, 40:10-15.

14. Bradley EL 3rd: A clinically based classification system for acute pancreatitis. Summary of the international symposium on acute pancreatitis, Atlanta, Ga, September 11 through 13, 1992. Arch Surg 1993, 128:586-590.

15. Liu C, Hung Y, Chuang Y, Chen YJ, Weng WS, Liu JS, Liang KY: Incorporating development stratification of Taiwan townships into sampling design of large scale health interview survey. $J$ Health Manag 2006, 4:1-22.

16. Charlson ME, Pompei $P$, Ales KL, Mackenzie CR: A new method of classifying prognostic comorbidity in longitudinal studies: development and validation. J Chronic Dis 1987, 40:373-383.

17. Deyo RA, Cherkin DC, Ciol MA: Adapting a clinical comorbidity index for use with ICD-9-CM administrative databases. J Clin Epidemiol 1992, 45:613-619.

18. Kuo JS, Ho YC: Board composition and community benefit expenditures of nonprofit hospitals. http://nccur.lib.nccu.edu.tw/handle/140.119/41790.

19. Hanley JA, Negassa A, Edwardes MD, Forrester JE: Statistical analysis of correlated data using generalized estimating equations: an orientation. Am J Epidemiol 2003, 157:364-375.

20. Flanders WD, DerSimonian R, Freedman DS: Interpretation of linear regression models that include transformations or interaction terms. Ann Epidemiol 1992, 2:735-744
21. Panageas KS, Schrag D, Riedel E, Bach PB, Begg CB: The effect of clustering of outcomes on the association of procedure volume and surgical outcomes. Ann Intern Med 2003, 139:658-665.

22. Birkmeyer JD, Dimick JB: Potential benefits of the new Leapfrog standards: effect of process and outcomes measures. Surgery 2004, 135:569-575.

23. Bureau of National Health Insurance, Department of Health, Executive Yuan. http://www.nhi.gov.tw/webdata/webdata.aspx? menu=17\&menu_id=661\&WD_ID=689\&webdata_id=3351.

doi:10.1186/1471-230X-12-112

Cite this article as: Shen et al:: The effect of hospital volume on patient outcomes in severe acute pancreatitis. BMC Gastroenterology 2012 12:112.

\section{Submit your next manuscript to BioMed Central and take full advantage of:}

- Convenient online submission

- Thorough peer review

- No space constraints or color figure charges

- Immediate publication on acceptance

- Inclusion in PubMed, CAS, Scopus and Google Scholar

- Research which is freely available for redistribution 